\title{
Role and Importance of Chemical Engineering in the Specialty Chemicals Industry - A Point of View from Process Development
}

\author{
Lukas Doulakas, Pascal Larpin, Horst Maire*, Laurent Menoud**
}

\begin{abstract}
Chemical engineers offer many skills perfectly in line with the requirements of the specialty chemicals industry, especially within process development. One of the main tasks of this department is to recruit and train young talent. A chemical engineer helps in defining new processes, improving established products and solving EHS (environment, health and safety) issues. Thus, the chemical engineer takes an active role in the main stages of a product life cycle and fosters through his communication skills cooperation with other colleagues working in different fields, such as production \& engineering, product management, technical services. In order to fulfill the industry requirements, the Higher Institutes of Technology are encouraged to continuously offer the best education in chemical engineering.
\end{abstract}

Keywords: Chemical engineering $\cdot$ Process development $\cdot$ Specialty chemicals $\cdot$ Young talent

\section{Introduction}

Even though the authors are working in process development groups within Ciba Specialty Chemicals Inc. the present article reflects our personal point of view and is based on our specific experiences. The opportunity to explain, particularly to young students, current industrial process development (PD) in a realistic way has been our main motivation.

${ }^{{ }^{*} \text { Correspondence: }}$ Dr. H. Maire Ciba Specialty Chemicals Inc.

Coating Effects Segment

Process Development Monthey; CE 6.23

Building WM-369B

Case postale

$\mathrm{CH}-1870$ Monthey

Tel.: + 41244744964

Fax: + 41244744979

E-Mail: horst.maire@cibasc.com

www.cibasc.com

${ }^{\star \star}$ Correspondence: Dr. L. Menoud

Ciba Specialty Chemicals Inc.

Home and Personal Care Segment

Process Development Monthey; HP 4.37

Building WM-374

Case postale

$\mathrm{CH}-1870$ Monthey

Tel: : 41244744052

Fax: + 41244744079

E-Mail: laurent.menoud@cibasc.com

www. cibasc.com
The basic questions, which finally lead to this publication, are:

- Do we need chemical engineers in the specialty chemicals industry?

- Is a process development group the right entry point for young chemical engineers?

- Do chemical engineers play an important role in industrial process development?

When considering all aspects, positive and negative, which currently influence and lead process development activities in the specialty chemicals industry, or what PD offers young talent, the answer to all the above questions is clearly yes!

One important task of PD is the recruitment and training of young talent and future managers. Assuming an ideal group size, where continuity and exchanges could be guaranteed, without affecting the necessary skills, a young graduate (male/female) will stay about 3-5 years in PD, where he/she receives and experiences his/her basic training in the company. After this period, which serves as preparation for their future career, they are well acquainted with products (chemistry, processes), production (plant, installation, equipment), working methodology, environment - health - safety (EHS) aspects and are well prepared to take over other responsibilities in the company.

The main objectives of a PD group are the development of inherently safe, reliable, reproducible, robust processes at low cost for new or existing products respecting quality constraints and legal EHS requirements. In order to fulfill this mission, PD groups consist often of an optimum balance between chemists and chemical engineers, where PD chemists or PD chemical engineers work in multiskilled teams, where the chemical engineer is the connecting link between technical (such as production \& engineering, quality control) and non-technical services (such as product management, marketing, product safety \& registration, research). To explain the role and importance of the process development, we chose the main stages of a product life cycle as a thread.

\section{Process Development and Product Life Cycle}

The different aspects of process development work could be discussed and illustrated in an exemplary way, when we look at the ideal product life cycle as shown in Fig. 1. It is obvions that not eve- 
ry product will follow this profile, but the development work must be made as if for an ideal product as described in the quality management $(\mathrm{QM})$ procedure. During the different phases in a product life, the role and the required knowledge will change with the problems encountered. In a general way, the product life can be subdivided in two main periods, which are separated by the launch of the product, or, more precisely, when the product passes to the responsibility of production. The subdivision in 'New Products' and 'Range Product' reflects precisely this fundamental difference. During the first period the product is fully integrated in the 'innovation process', where teams (often headed by marketing or R\&D) consider all questions and problems to be solved up to the launch. On the other hand in the second period, when the product becomes a range product, production takes over the responsibility. Let us have a look now at what happens typically during the above-mentioned phases.

\subsection{Development \& Introduction}

When considering the features in this phase, it is obvions that quantities are rather small: before the first introduction, only lab or pilot samples are available. When the process development is involved, research work is almost completed and the chemistry is more or less defined. Following the decision based on a business justification to bring such a product to the market, the registration of the molecule will start and, for products foreseen to be used in 'food contact', application approvals are needed (e.g. FDA, GMP, FPL). Project teams will be set up, composed of colleagues from research, product management, marketing, registration, production \& engineering, quality control and process development. In order to bring the product to the market as quickly as possible (catchword: 'faster time to market') we are asked to accept compromises: first production campaigns will take place in existing facilities, which are often not completely suitable for our chemistry; our process is not fully optimized, EHS problems are only solved in a semi-optimal way (such as incineration of mother liquors instead of solvent recovery), production costs are still too high. There remains a large potential for process optimization.

Fig. 2. Development of volume, sales price and 'total production costs (TPC)'

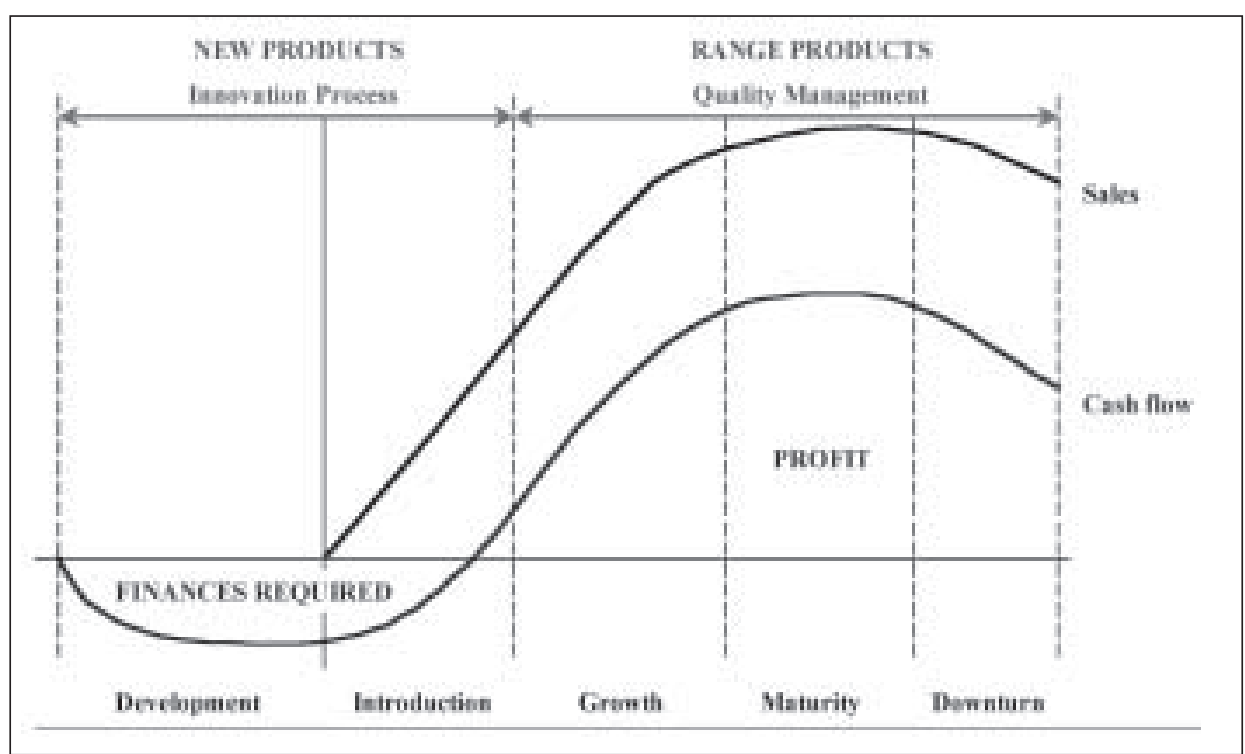

Fig. 1. Sales and cash flow during the product life cycle

\subsection{Growth}

Once on the market, the product enters into competition with existing products. If our perception of the advantages (and disadvantages) of our product, the positioning and the marketing strategy were correctly made, the sales will grow more or less rapidly. Then it becomes increasingly important that the margin (simply said the difference between sales price and total production costs) in Fig. 2 is maximum and enough capacity is available; in addition increasing EHS problems must have been solved. Supposing the company benefit is simply the product of margin multiplied by volume (sold quantities), the process development in collaboration with production \& engineering can only affect the benefit by improving the margin, thus lowering the production costs. And you can assume that the sales price will decrease all the time. If we have the ideal case, that our product is still protected by international patents, the most important task of the process development group is to guarantee the lowest production costs possible in order to remain competitive and to raise the hurdle for our competitors after the patent protection has expired.

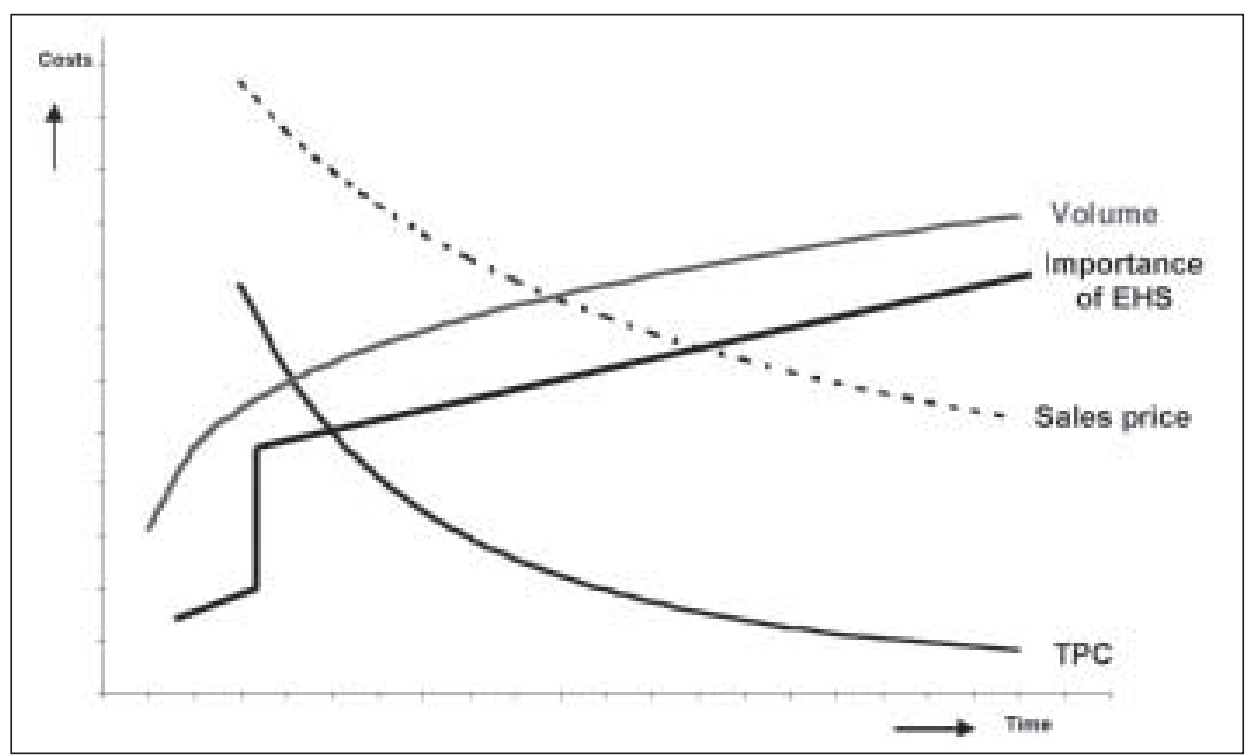




\subsection{Maturity}

If a product reaches this phase of its life, we are often confronted with a very curious situation. The whole process (including solvent recovery units, waste gas treatment) has been optimized over a number of years. Specific skills and knowledge have been built up and documented. The quality is under control and the production costs are (sufficiently) low. It is normal that the process development decreases or stops its activities related to this product. When entering this phase, the process development group 'loses' more and more specific product know-how. The main reasons are that development is now working on other products; PD must also face a high rate of personal fluctuation (especially with the chemists and chemical engineers) and unfortunately a reduced personal strength in a difficult economic situation.

In addition, the production staff might have realized a number of minor process changes to solve specific (everyday) problems, which could lead, at least, to a different or modified process. All together we could end up with process that is out of control, where the production is no longer able to deliver the product on time within the required quality and quantity. To counteract such a non-desired evolution, process development groups must be close to production (especially in key production sites), with a group size which enables us to do our work in a suitable way and finally an active personal recruitment is necessary to anticipate future personal changes.

\subsection{Downturn}

Now the last phase is entered, where further cost reduction becomes nearly impossible, if PD has correctly done its homework up until now. The focus of development work becomes more and more concentrated on the last remaining potentials for cost reduction. The company might need to accept higher risks in terms of quality; e.g. less quality control could be acceptable, because the process should be under control. However, higher risks in terms of safety will never be accepted!

In order to avoid or to prevent the undesired downturn of sales, R\&D and product management try to add specific features to the product, which could be of interest for our customers, e.g. special sales forms trying to re-start a new cycle of life.

\section{The Role of Process Development within the Different Phases in a Product Life}

\subsection{New Products}

At this early stage in product life, the development work is best comparable to the research work at the university. To follow a product from its birth (first trials in lab, then pilot), over the project work (e.g. definition of product properties, process optimization, identification of relevant parameters, reaction kinetics, preparation of launch), until the first batch is in production creates in most cases a very exciting and satisfying job. The chemical engineer must build up specific knowledge about the product and the process, and a lot of open questions need to be answered.

Once a market justification is established for the product, marketing will put on pressure in order to have this product manufactured under 'real' conditions on a production scale. One of the crucial problems during this first introduction is to match the defined quality, which must be representative of the future requirements. Nevertheless, quality deviations are quite normal at this early stage and we will follow the 'learning curve' as quickly as complete has been our former development work. This means that we need an integral understanding of parameters which may influence the product quality especially after a scale-up. It is worth mentioning at this point that during a scale-up, not all physical effects like mixing (micro, macro, time), heat transfer, shear stresses can be kept constant at the same time. We need to know what the most important effects are. And quite frequently we have to find the right compromise between two or more parameters. Finally in such cases, chemical engineers have to finalize the optimization procedure, such as stirrer speed, on the production scale.

Due to small initial volumes, it is evident that the required production capacity is low, and therefore problems relevant to EHS are smaller, but never zero. The project team will or may accept non-optimal solutions in order to accelerate the market launch. But to avoid misunderstandings: legal EHS constraints must always be observed and for safety aspects, the Company will never accept a compromise! To strive for the highest safety standards is part of our philosophy.

For new products, the role of chemical engineer is to develop a reproducible and cost competitive process and edit the manufacturing procedure. As part of this document, a complete risk analysis must be carried out, which is time-consuming and is always a special task performed in groups consisting of representatives from production and process development and other specialists such as safety, mechanical and electrical engineers. Because timetables are always ambitious, this time must be taken into consideration on the schedule. From the chemical engineer, a clear picture and knowledge of the new process is needed, as every last minute process or technology change could affect the risk analysis! The chemical engineer must apply the best methodology as economical and time constraints tend to skip the pilot phase, thus introducing a lab process directly into fullscale production.

Besides, an understanding of nonchemical processes is not only desirable but also indispensable. Chemical engineers may have slight advantages in such cases because of their wide-ranging education compared to pure chemists, even if we clearly need both of them in the process development.

Although the product is in a very early phase, some of the future work to be done will be already defined and identified at this stage. The process choice with the definition of raw materials, solvents and specific technologies defines at this stage potential future problems such as supply problems with 'exotic' raw materials, waste gas treatment, solvent recovery of complex mixtures, control of special technologies and disposal of solid waste. Such problems, and this is only a small selection, become more and more important when larger quantities must be produced. This explains clearly the importance of the role of chemical engineering and of the process development work at this stage.

Another crucial point is the identification and anticipation of mid- or long-term requirements. Decisions have to be made frequently on the marketing forecast, which is based on a multitude of assumptions. This might lead to an investment in a period where the (cumulated) profit is still negative.

\subsection{Range Products}

\subsubsection{Improved Processes}

For a product in the mature part of its life cycle, the responsibility is then handed over to production staff. However our colleagues still need team support from PD in order to optimize the process and decrease production costs. In the growth phase of the product life cycle, the chemical engineer helps in decreasing variable 
costs (raw materials) by reducing the excess reactant and increasing the yield, using among other methods incremental step improvements (EVOP), factorial plan, and basic reaction engineering techniques. Often, due to the volume effects, small incremental improvements could help save several hundred thousand CHF. Such productivity improvements are always possible and not in contradiction with a high-quality development during launch phase. The latitude for optimization and debottlenecking is mainly within the domain of scientific and technical innovation, such as new catalyst discovery, better solvent and catalyst regeneration, and new technology such as membranes. New computer technologies such as process simulation and advanced modeling also allow further improvements on product cost.

With regard to fixed costs, the role of $\mathrm{PD}$ is to apply chemical engineering to specific fields such as decreasing energy consumption, solving EHS issues and reducing waste production. A special focus is given to solvent recovery units, as they deal with both energy and waste.

An interesting fact to point out is that incremental improvements made directly by the production over several years often lead to reduced process control (correlating with quality problems), because the process could be now be different (introduction of 'process change procedure'). In this case the chemical engineer is asked to re-invent the wheel (that means going back to the former process). Communication skills are necessary to find out the history behind the problem.

\subsubsection{Capacity}

Following process optimization aimed at increasing productivity, the sales could (should) reach such a volume that no more idle capacity is available in production; so further growth will only be possible after a substantial investment.

With this positive outlook of growing sales, PD plays an important role as process owner. On the one hand, by offering the sum of expertise and know-how gained during the launch and growth phase, including statistical process analysis and feedback from past mistakes. On the other hand, after the analysis of the situation and if latitude is given, time for innovation is open. As a member of a project team, the chemical engineer will cover fields such as process design, reaction technology, and new process technologies. Finally, based on previous experience and innovation in case of op- timum process set-up, a choice must be made between either debottlenecking existing equipment (more or less status quo for the process) or purchasing new equipment allowing the ideal process to be reached. One of the challenges the chemical engineer and the whole team have to face is parallel work on running production.

\subsubsection{Environment, Health, and Safety}

Together with capacity projects, the importance of EHS problems is proportional to production quantity; that means, for mature products with big production volumes, specific and high-tech solutions are usually needed to improve the ecoefficiency. In order to respect and even anticipate regulations, EHS equipment is very often associated with investment (see the step in EHS level in Fig. 2), where a definitive and efficient EHS solution must be available. As process owner, the chemical engineer participates in designing the unit, followed by start-up and handover to production. The task of continuously reducing the environmental impact of our products and manufacturing procedures is certainly a key element and one that definitely requires better support during the education of young chemical engineers.

\subsubsection{Energy and Raw Materials}

In direct connection with EHS aspects, energy consumption and waste are of high interest to decrease cost. Especially within Ciba Specialty Chemicals, a strong focus is presently put to both topics, as the company has established ambitious goals to decrease the energy and raw material consumption - assuming that less raw materials translates to less waste. In collaboration with PD, production \& engineering have initiated actions to encourage day-to-day actions (better utilities management) and in parallel identifying the big consumers and express the energy efficiency unit per unit. For a mono-product plant, the cost of steam is very often the highest cost block, followed by electricity and cooling water. Overall and specific energy balances are set up to identify potential fields for improvement. For example, applying techniques such as pinch technology helped to decrease the energy consumption. As steam is the major block cost, in a second example, we developed a specific tool to measure the steam consumption on-line and indirectly through condensate flow. Using this tool, we were able to identify more than $90 \%$ of steam consumers. Then comparing the theoretical demand with the effective requirement, we put in evidence the biggest energy consumers (losses higher than 15\%) such as solvent recovery units and continuous working unit operation.

\section{Chemical Engineer: Role and Perspective}

Our description of the role of the chemical engineer in specialty chemicals should help young talent to better understand our needs. Nevertheless, whenever discussions with students are undertaken, one question is regularly asked: 'What is the role and the future of a chemical engineer in industry?' Many reasons may be hidden behind this question such as lack of industrial knowledge, uncertainty, fear, accidents in industrial plants, mergers and re-organization. Whatever the reasons are, a lack of communication persists between the chemical industry and universities: let us take the opportunity to give the point of view of chemical engineers who asked the same questions a few years ago.

\subsection{General Outlook}

All engineers employ mathematics, physics, and engineering skills to overcome technical problems in a safe and economical fashion. Yet, it is the chemical engineer alone that draws upon the vast and powerful science of chemistry to solve problems. He uses computer technology to optimize all phases of the product life cycle and therefore needs to understand how to apply computer skills. $\mathrm{He}$ designs equipment and develops processes for large-scale chemical manufacturing, plans and tests methods for manufacturing the products and treating the by-products, and supervises production. The studies at technology universities help him to acquire all the knowledge necessary for these tasks. The wide range of courses on offer covering mass, momentum and energy balance, thermodynamics and chemical kinetics are well adapted to requirements of industry.

However, a chemical engineer in the industry is mainly occupied with finding appropriate solutions even under difficult conditions (cost and time restrictions, different languages, safety policy etc.). This aspect and the responsibility to take decisions are the main differences between industry and university. 


\subsection{The Chemical Engineer as Universal Engineer}

Many companies are under great pressure. The competition never sleeps, and it is no secret that high-quality products at aggressive prices originating mainly from Asia permanently attack the worldwide position of Swiss (or European) companies. Constant high-quality products, innovation and cost reduction are some of the best answers to this problem. The chemical engineer helps in all three domains and faces therefore many of the same challenges that other professionals face. Despite a title that suggests a profession of narrow specialists, in fact chemical engineers must be extremely open-minded and have to be able to handle a wide range of technical problems. One of his/her prime skills and responsibilities, however, is that of the cost reduction.

Under cost reduction, we do not only refer to the classical field of optimization of older products by improving their conversion, saving energy or decreasing waste. But also cost reduction already starts when launching a new product. Existing capacities have to be utilized instead of building new facilities or buying brand-new equipment. It is the art of the chemical engineer to propose the best and most economic option.

Another prime skill lies in the field of production. Knowledge of the different unit operations (e.g. distillation, heat exchange, filtration) is important for the global understanding of the production. Finally, a chemical engineer is predestinated for all works where balances (e.g. mass, energy or cost balance) have to be calculated.

Nevertheless, the wide range of the problems that a chemical engineer has to solve may rapidly require more than his knowledge and experience. Therefore, challenging the problems with other colleagues is part of his daily work. His skill in understanding different fields without always being a specialist, often provides the opportunity to run the organisational part of a project.

\subsection{Environment, Health, and Safety}

A very important point is the safety culture and the protection of the workers and of the environment. Many industrial plants are located close to civil populations. The future of the chemical industry can only be guaranteed by running safe processes with low emission impact on the environment coupled with appropriate hygiene standards. A chemical engi- neer spends a lot of time providing economical answers to increase the safety of installations, clean-up yesterday's waste and prevent tomorrow's pollution. The working methods in the field of EHS follow well-defined rules and require rigorous methodology. The first part of this work consists of collecting safety data. The second part consists of detecting and elaborating dangerous situations like risk of explosion, runaway scenarios etc. Finally, finding and introducing appropriate solutions to minimize the risk (risk = probability $\times$ severity) is the last step before a process can be run.

\section{Future Prospects for a Chemical Engineer}

Following the main stages of the product life cycle, the tasks of a chemical engineer are numerous, as numerous as the number of partners within an international company. Thanks to his education in an institute of technology such as $\mathrm{ETH} / \mathrm{EPF}$, there exists little doubt concerning the future prospects of the chemical engineer. The classical opportunities in process development are not the only domain for interesting jobs, but commonly used as a starting point in the specialty chemicals industry. After a few years, strategic positions like production, product management, technical marketing, and investment project management are open for the acquisition of further experience. Many chemical engineers have found their way into upper management. Outside the chemical industry, the chemical engineer's expertise is also applied in the areas of law, education, publishing, finance, and medicine, as well as in many other fields requiring technical experience.

Unfortunately, chemical engineering graduates may also face competition for jobs; however, the possibility of practical training exists and contacts with people from industry surely help to find jobs even under difficult transient situations. Rest assured, the specialty chemicals industry will always offer interesting opportunities for young talent!

Received: February 19, 2002 